\title{
Free-Drawing from Memory in Constructional Apraxia: A Case Series
}

\author{
Simon M. McCrea \\ Independent Consultant, Regina, Saskatchewan, Canada
}

Email address:

simon.mccrea@usask.ca

\section{To cite this article:}

Simon M. McCrea, Free-Drawing from Memory in Constructional Apraxia: A Case Series. American Journal of Psychiatry and Neuroscience. Vol. 3, No. 6, 2015, pp. 125-141. doi: 10.11648/j.ajpn.20150306.15

\begin{abstract}
Constructional apraxia is a neuropsychological and neurological impairment in activities such as building, assembling, and drawing. In this study data is presented on the performance of left and right hemisphere single focal stroke lesion participants on drawing tasks of houses, trees, and persons. Forty-one participants completed a comprehensive neuropsychological battery including the house-tree-person drawing task. The drawings were then rated as exhibiting signs of neurological impairment or non-impairment by three evaluators without knowledge of the medical condition of the patient. There was $72 \%$ agreement between the three evaluators of the 123 drawings. Both an analysis of variance and case series analysis was used to examine the data. Patterns of concordance with a previously outlined detailed neuropsychological model of constructional apraxia were found and the implications of the results are analyzed in the context of the current neuropsychological literature.
\end{abstract}

Keywords: Constructional Apraxia, Free-Drawing, House-Tree-Person Drawing Task, Drawing-from-Memory, Unilateral Cortical Lesions, Stroke, Visual Imagery, Lexicosemantics

\section{Introduction}

Human drawings of real-life animal, plant and partial human figures is an ancient ability or pursuit of Paleolithic man and woman. One of the best examples of this is the Chauvet caves of southern France dating to 32,000 years old of the Aurignacian period. This cave which was discovered in 1994 is now a UNESCO world heritage site. Pictures of now extinct wooly rhinoceros, bison, cave lions, panthers, bears and cave hyenas along with an incomplete female Venus figure and abstract line and dot markings mark these caves. Paintings and drawings on the walls depict distinctive movement of animal herds jostling with their horns, and even whole visual scenes suggest some sense of contemporary visual perspective-taking [1]. Human beings have clearly placed a premium on visual artistic abilities for thousands of generations. Despite the importance of visual artistic abilities, little is known about the neural correlates of them and much work is yet to be done to understand the intrinsic nature of aesthetic appreciation for art [2]. What little research on these drawing abilities currently exists suggests that these neural networks are widespread and distributed across the brain, complex and consisting of multiple modules working in a coordinated manner.
In neuropsychology the study of drawings has almost exclusively been done in the context of studying the copying of abstract figures that can be objectively scored but that lack lexical and semantic associates of real animate objects. There are many reasons why abstract drawings from a theoretical perspective would not be expected to tap the same neural modules of rich contextually alive visual perspective. There may be some way out of this apparent objectivism versus subjectivism clash of underlying epistemologies within this domain [3]. For instance, in neuropsychology case-series methods can usefully complement single subject designs and techniques [4]. A mixed-methods approach then using detailed studies of individual cases and overall linkage across cases in terms of: (i) multiple convergent sources of evidence, (ii) creation of a common case series database and (iii) maintenance of a continuing chains of evidence can assist in case study triangulation [5]. And luckily study of drawings is not without an extensive empirical and theoretical basis in clinical psychology.

In personality research drawings of persons have been used to measure intelligence since the turn of the last century [6-9]. Thus, a voluminous empirical database on administering, scoring and interpreting standardized 
drawings already exits. Neuropsychological tests of human figure drawings have been developed to evaluate visuoconstructive and self-perception across the entire human age span [10, 11]. Moreover, many neuropsychiatric disorders have disturbances in body representations [12] and can be readily assessed directly by drawings of human figures in conjunction with other clinical information and case history [13]. After the Wechsler scales began to displace such drawing tests for the appraisal of intelligence in the 1940's [14], drawings continued to be used widely in clinical practice for the assessment of personality and purported unconscious processes $[15,16]$.

There is good clinical evidence of the usefulness of human drawings. A 2001 study of 82 directors of APA doctoral programs in clinical psychology indicated that training in the use of human figure drawings were rated as "required" in $37 \%$ of those polled [17]. This rate of usefulness was the third most popular only to the Rorschach (80\%) and Thematic Apperception Test (70\%). In a large sample of 1500 clinical psychologists surveyed at random from American Psychological Association databases, the housetree-person drawing test was ranked eighth in popularity behind the Rorschach (fourth), Bender Visual Motor Gestalt Test (fifth), and the Thematic Apperception Test (sixth) among projective techniques [18,19]. In this same study Camara and colleagues (2002) found that the house-treeperson drawing task was used on a regular basis by $37 \%$ of clinical psychologists and $18 \%$ of clinical neuropsychologists [20].

As research began to accumulate on the use of the housetree-person for diagnosing and understanding personality, it became increasingly mainstream as part of a complete diagnostic evaluation in clinical psychology. However, the house-tree-person test has been much maligned in recent years for its lack of statistical reliability, inherent subjectivity and low concurrent validity with other personality instruments. It has now been deemed inadmissible in most United States courts [21]. It cannot be used solely as a single indicator of any diagnostic entity. Nonetheless, with these caveats aside the house-tree-person drawing task continues to be widely used in clinical evaluation projective portfolios for the purpose of developing hypotheses and psychodiagnostic inferences. Incidentally, the house-tree-person drawing test has long been known to be a sensitive test of organic impairment, and along with the Wechsler scales, was among the first instruments to be used for such purposes [7-9].

In sum, there is an extensive published empirical literature on the house-tree-person projective drawing test usage in patients with brain lesions [22-28]. If researchers wanted to study the lexical and semantic processes that are involved free-drawing, then it could be argued that there would not likely be a more appropriate sets of tests to select for such purposes. Under such circumstances the use of the housetree-person test could be a natural complement to a selected set of neuropsychological tasks dependent upon patient characteristics and the nature of the clinical question. In this study the neural correlates of constructional apraxia in a drawing-from-memory test were examined with house-treedrawing test.

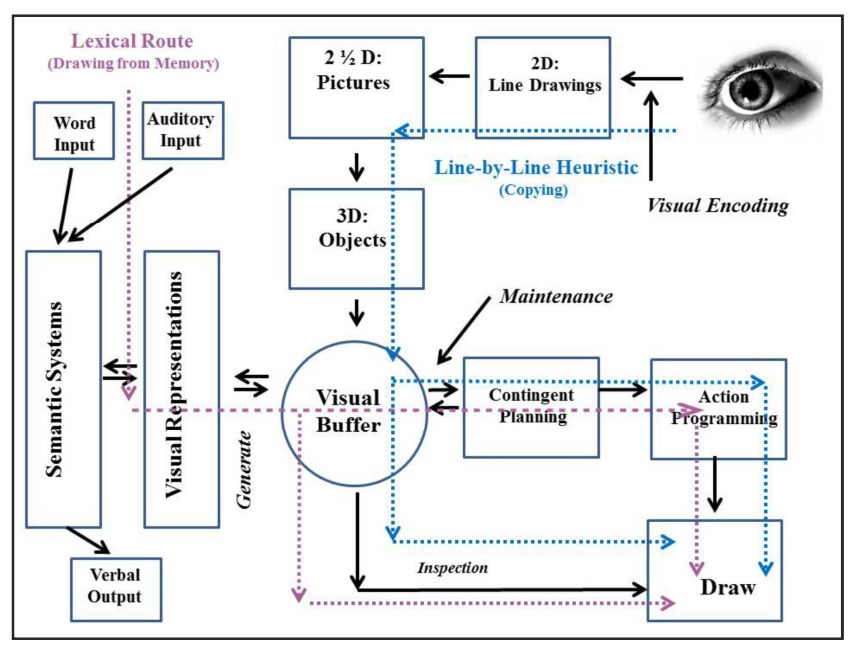

Figure 1. Contemporary model of constructional apraxia-related free drawing incorporating the lexical and line-by-line heuristic routes. Cognitive neuropsychological model illustrating Trojano and Grossi's (1994) lexical route involved in drawing from memory (purple) and line-by-line heuristic used in copying from a model (blue) [99]. Adapted from Figure 4 on page 69 of Ref. [29].

Constructional apraxia as applied to "free drawing" or "drawing-from-memory" has previously been the subject of an extensive theoretical review [29] and Figure 1 above based on that review depicts a contemporary model of constructional apraxia based on the most widely accepted models of the disorder studied from different perspectives. That review discusses the history of use of drawing tests, neuropharmacological studies with drawing tests, psychopathology, history of the study of constructional apraxia, constructional apraxia and mental imagery, syndromes co-occurring with constructional apraxia, modeling of the cortical interactions in free-drawing and the neuroimaging of drawing-related constructional apraxia. Furthermore, a previously published article discusses the ANOVA of the main effects and interactions of this drawingfrom memory in stroke-induced constructional apraxia in this data set [30]. In this study we will examine this data set in detailed case series with qualitative presentation of the drawings along with an examination of a case series.

\section{Method}

\subsection{Institutional Review}

In this study forty-one participants with: (i) focal singular contiguous cortical stroke lesions, (ii) mixed multiple cortical or subcortical lesions, or (iii) general adult clinical neuropsychology cases were referred for neuropsychological evaluations. Participants were recruited into the study over a two-year period at the Wascana Rehabilitation Centre in Regina, Canada. Ethical consent for the study to proceed was granted by Research and Performance Support of the Regina Qu'Appelle Health 
Region in 2010. All participants were informed about the potential inclusion of their data into studies and signed an informed consent form prior to participation. Patients were assured that any data included in such studies would be deidentified to safeguard participant's confidentiality and anonymity. This research study adhered to the principles declared in the Canadian Tri-Council Policy Statement: Ethical Conduct for Research Involving Humans [31] as well as with the principles stated in the Declaration of Helsinki [32]. Parts of the method for this study were examined previously [30]. A completely new presentation of the data set are presented herein.

\subsection{Sample Characteristics}

The sample consisted of 28 men and 13 women and thus there was a disproportionate sampling of the sexes $\left[\chi^{2}=\right.$ 22.5, $\mathrm{p}<0.0001]$. Every effort was made to have proportional representation of the sexes. Men do outnumber women in acute stroke units and brain injury centers at younger ages. Reasons for the disproportionate sampling have previously been shown to be due to a number of interacting factors. Men have an elevated risk of cerebrovascular accident at all ages [33]. At the relatively younger ages of stroke participants recruited into this study, testable stroke participants are often male. Younger female stroke participants tend to have poorer prognosis due a selection effect of stroke severity and etiology [34]. It has been long known that younger men have disproportionately higher rates of acquired brain injury [35]. The elevated rates of men with stroke and acquired brain injury would in all likelihood largely account for this discrepancy between the sexes.

Table 1. Demographics related to sex, handedness, age and education.

\begin{tabular}{lllllllll}
\hline $\begin{array}{l}\text { Drawing } \\
\text { group }\end{array}$ & M & F & \multicolumn{2}{c}{ Handedness } & Age & \multicolumn{3}{c}{ Education } \\
\hline & & & RT & LT & Avg & SD & Avg & SD \\
\hline Normal & 16 & 10 & 22 & 4 & 44 & 15 & 10 & 6.3 \\
Impaired & 12 & 3 & 13 & 2 & 39 & 17 & 11 & 5.0 \\
Total & 28 & 13 & 35 & 6 & 42 & 15 & 10 & 5.8 \\
\hline
\end{tabular}

Six of 41 participants were left-handed constituting $14 \%$ of the sample. Hardyck and Petrinovich's (1977) review suggest that approximately $10 \%$ of the general population is lefthanded [36]. A chi-square test found this difference nonsignificant $(p=0.23)$. This result suggests that handedness in participants will not likely skew expected distributions of test scores in the sample so as to confound the results and generalizability of the findings - (see Table 1). The mean age of the sample was 42 years $(\mathrm{SD}=15)$, and there was difference in age between the normal and impaired groups $[\mathrm{F}(1,39)=0.83, \mathrm{p}=0.37]$. The group as a whole was less educated than the general population (mean $=10$ years, $\mathrm{SD}=$ 5.8 ), however there was no difference in education levels between the normal and impaired groups $[F(1,39)=0.08, p$ $=0.78]$.

The premorbid FSIQ as estimated using the Advanced
Clinical Solutions Test of Premorbid Functioning [37] was in the average range for the group as a whole (mean $=102, \mathrm{SD}$ $=13$ ). Importantly, there were no differences in premorbid levels of general intelligence between the normal and impaired groups $[\mathrm{F}(1,39)=1.72, \mathrm{p}=0.20]$. Participants were evaluated an average of 16 months $(\mathrm{SD}=22)$ post-injury. This is a safe duration post-injury to assess patients [35]. There were no differences in the number of months postinjury that normal and impaired participants were tested $[\mathrm{F}(1$, $33)=3.58, p=0.70]$. Participants would have experienced near maximal levels of functional recovery at 16 months post-injury [35]. Previous studies of constructionally-apraxic (CA) patients have only tested patients in the post-acute phase (first few weeks after stroke) in the neurological ward before discharge [38]. At such short durations neuropsychological impairment may be due to non-specific and indirect effects of the lesion (e.g., general cognitive slowing or diaschisis) rather than being a function of specific localized modular damage [39]. This is a potential confound that was avoided in the current study.

\subsection{Cross-Tabulations of Etiology and Constructional Apraxia Status}

A chi-square analysis of the nominal variables of etiology crossed with impaired/non-impaired status was nonsignificant $\left[\chi^{2}(5)=4.84, p=0.44\right]$. A chi-square analysis of the nominal variables of anterior/posterior localization of lesion and impaired/non-impaired status was similarly nonsignificant $\left[\chi^{2}(2)=3.73, p=0.16\right]$. A chi-square analysis of the nominal variable of left/right localization of lesion and impaired/non-impaired status was also non-significant $\left[\chi^{2}(2)\right.$ $=3.73, \mathrm{p}=0.16]-($ see Table 2$)$. The results of the analysis of nominal and continuous variables suggested that drawingimpaired and drawing-normal subject groups were comparable (Table 2). Although the present study constitutes a small sample it is among the largest published of qualitative case presentations of $\mathrm{CA}$ patients compared to previous studies [38].

Table 2. Laterality, anterior-posterior lesion site, and etiology.

\begin{tabular}{|c|c|c|c|c|c|c|c|c|c|c|c|c|}
\hline \multirow[t]{2}{*}{$\begin{array}{l}\text { Drawing } \\
\text { group }\end{array}$} & \multicolumn{3}{|c|}{ Laterality } & \multicolumn{3}{|c|}{$\begin{array}{l}\text { Anterior- } \\
\text { Posterior }\end{array}$} & \multicolumn{2}{|c|}{ Etiology } & \multirow[b]{2}{*}{3} & \multirow[b]{2}{*}{4} & \multirow[b]{2}{*}{5} & \multirow[b]{2}{*}{6} \\
\hline & $\mathrm{L}$ & $\mathrm{R}$ & - & A & $\mathrm{P}$ & - & 1 & 2 & & & & \\
\hline Normal & 4 & 2 & 20 & 4 & 2 & 20 & 9 & 7 & 4 & 2 & 2 & 2 \\
\hline Impaired & 2 & 5 & 8 & 2 & 5 & 8 & 7 & 7 & - & 1 & - & - \\
\hline Total & 6 & 7 & 28 & 6 & 7 & 28 & 16 & 14 & 4 & 3 & 2 & 2 \\
\hline \multicolumn{13}{|c|}{$\begin{array}{l}\text { Normal }=\text { non-constructionally apraxic participants; Impaired }= \\
\text { constructionally apraxic participants; } \mathrm{L}=\text { left hemisphere, } \mathrm{R}=\text { right } \\
\text { hemisphere; } \mathrm{A}=\text { lesion anterior to the central sulcus; } \mathrm{P}=\text { lesion posterior to } \\
\text { the central sulcus; } 1=\text { motor vehicle accident and diffuse injury; } 2=\text { stroke; } \\
3=\text { general neuropsychology participants; } 4=\text { subcortical etiology; } 5= \\
\text { personality disorder; } 6=\text { concussion. }\end{array}$} \\
\hline
\end{tabular}

\subsection{Constructional Apraxia Rating System}

Participants with single focal contiguous stroke lesions of an ischemic or hemorrhagic nature or neurosurgical resections in 
the left or right hemisphere were the primary focus of the investigation. Strokes and neurosurgical resections are among some of the best natural means of establishing brain/behavior relationships in neuropsychological studies [40]. Participants were determined to belong to a neurologically impaired or normal group by a combination of two rating systems which were subsequently demonstrated to show near equivalence. In the first rating system, (Unanimous Rating System) those participants demonstrating complete agreement across three trained raters on at least one drawing $\{(1)$ house or (1) tree or (1) person $\}$ ] were classed as impaired regardless of the status of the other two drawings in the set of 3 . Again, there are 41 participants with three drawings each for a total of 123 drawings. The 123 drawings were first randomized and then rated blindly by three raters. These drawing evaluators did not administer any neuropsychological tests to the participants and thus were not biased by experimenter expectancy effects [41]. Nor did these raters have any knowledge of lesion grouping of the patients. Previous studies have included novice raters who did not have any detailed neuropsychological knowledge of CA and yet subsequently satisfactorily scored participants' drawings [42]. Sixteen participants were classified as impaired using the first rating system. In fact using a short training program consisting of directed readings on visual indicators of brain damage in drawings trainees were reliably able to determine impaired versus normal non-CA drawings significantly above chance - see next section for a forthcoming discussion of reliability.

In the second rating system, (Threshold Rating System) those participants who scored above critical cutoff were deemed impaired (e.g., 12 on an 18 point scale). In the second rating system each house, tree, and person drawing was either dichotomously rated by three raters as either normal ( 1 point) or impaired ( 2 points). This rating system provided for a total of 9 possible ratings collapsed across the three raters for each of the individual forty-one study participants. Therefore, a minimum possible score for an individual subject was 9 and a maximum was 18 .
Importantly, using the second rating system, 15 participants were classed as impaired. Using both rating systems together 14 participants in common were classed as impaired. One subject from the Threshold Rating System was deleted because this person did not complete essential neuropsychological tests and a second subject was deleted from the Unanimous Rating System because one of the drawings in a set was unscorable.

One new subject was added to the second Threshold Rating System impaired group. This additional subject, who had a right cerebellar lesion, demonstrated no unanimous impairment across any individual drawings but did show significant levels of cumulative impairment across all drawings (score $=14 / 18)$. Finally, one subject was deleted from the Threshold Rating System group because of a nonrelevant nonverbal learning disability. There was 93\% agreement between the Unanimous Rating System and Threshold Rating Systems categorization of impaired and normal participants. Henceforth the second Threshold Rating System was used for all of the subsequent analyses as it was subsequently found to provide the most robust measure of overall sensitivity to constructional apraxia in its diverse forms.

Theoretically the Threshold Rating System should provide more robust measure of within-subjects or repeated measures means of identifying a drawing non-specific type of constructional apraxia. The Threshold Rating System thereby should ideally maximally partition out error variance due to individual differences crossed with type of drawing. Additionally, the second Threshold Rating System has a content or visual stimulus degree of nonspecificity to all three drawing tasks in comparison to the first Unanimous Rating System. The neuropsychological examiner of the 41 individual patients did not perform any of the constructional apraxia ratings. The ratings of the drawings were performed by two volunteer Ph.D. trained clinical psychologists and a graduate student in clinical psychology. For a complete description of the sample of participants refer to Table 3.

Table 3. Description of individual case's lesion, etiology and demographics.

\begin{tabular}{|c|c|c|c|c|c|c|c|c|c|}
\hline Case & Lesion and Etiology & Lat. & $\mathbf{A} / \mathbf{P}$ & Sex & Age & Hand & Educ & Rating & DAP Score \\
\hline $1 \mathrm{~A}$ & RT ant TL, insula \& basal ganglia & Right & Ant. & $\mathrm{M}$ & 56 & Right & 16 & 15 & 92 \\
\hline $2 \mathrm{~A}$ & RT. basal ganglia \& insula stroke & Right & Ant. & M & 64 & Left & 14 & 18 & 59 \\
\hline $3 \mathrm{~A}$ & RT pos mid TG \& AG \& pos SPL & Right & Pos. & M & 35 & Right & 12 & 12 & 102 \\
\hline $4 \mathrm{~A}$ & Right temporoparietal resection & Right & Pos. & M & 40 & Left & 14 & 16 & 107 \\
\hline $5 \mathrm{~A}$ & Right temporoparietal stroke & Right & Pos. & M & 56 & Right & 16 & 17 & 95 \\
\hline $6 \mathrm{~A}$ & Left pos. temporal lobe stroke & Left & Pos. & $\mathrm{F}$ & 41 & Right & 12 & 14 & 101 \\
\hline $8 \mathrm{~A}$ & MVA and diffuse injury & - & - & M & 16 & Right & 10 & 12 & 116 \\
\hline $9 \mathrm{~A}$ & MVA and diffuse injury & - & - & M & 50 & Right & 10 & 12 & 99 \\
\hline $10 \mathrm{~A}$ & RT. superior cerebellar stroke & - & - & M & 57 & Right & 16 & 14 & 95 \\
\hline $11 \mathrm{~A}$ & Assault and diffuse injury & - & - & $\mathrm{F}$ & 17 & Right & 10 & 15 & 106 \\
\hline $12 \mathrm{~A}$ & MVA and diffuse injury & - & - & M & 17 & Right & 11 & 15 & 92 \\
\hline $13 \mathrm{~A}$ & MVA and diffuse injury & - & - & M & 16 & Right & 10 & 15 & 75 \\
\hline $14 \mathrm{~A}$ & Bilateral thalamic infarcts & - & - & M & 28 & Right & 17 & 16 & 81 \\
\hline $15 \mathrm{~A}$ & Cardiac arrest and global anoxia & - & - & M & 50 & Right & 14 & 17 & 95 \\
\hline 1B & Left frontal lobe stroke & Left & Ant. & $\mathrm{F}$ & 56 & Right & 14 & 10 & 106 \\
\hline 2B & Left inferior frontal lobe stroke & Left & Ant. & M & 64 & Right & 17 & 11 & 120 \\
\hline
\end{tabular}




\begin{tabular}{llllllllll}
\hline Case & Lesion and Etiology & Lat. & A/P & Sex & Age & Hand & Educ & Rating & DAP Score \\
\hline 3B & Left parietal lobe stroke & Left & Pos. & F & 47 & Left & 16 & 9 & 109 \\
4B & Left inferior temporal lobe stroke & Left & Pos. & F & 63 & Right & 12 & 11 & 92 \\
5B & RT ant inf TL and rt orbital gyrus & Right & Ant. & F & 35 & Right & 17 & 9 & 90 \\
6B & RT inferior \& middle frontal gyrus & Right & Ant. & M & 55 & Right & 16 & 10 & 99 \\
7B & Fall and diffuse contusions & - & - & M & 46 & Right & 9 & - & - \\
8B & West Nile virus encephalitis & - & - & M & 47 & Right & 19 & 9 & 116 \\
9B & Personality Disorder & - & - & M & 31 & Right & 13 & 9 & 112 \\
10B & MVA and diffuse injury & - & - & M & 18 & Right & 12 & 9 & 116 \\
11B & MVA and diffuse injury & - & - & M & 37 & Right & 18 & 9 & 105 \\
12B & Assault and diffuse injury & - & - & M & 26 & Left & 10 & 9 & 113 \\
13B & Right pos. thalamic infarct & - & - & M & 23 & Right & 16 & 9 & 102 \\
14B & Fall and diffuse injury & - & - & M & 61 & Right & 19 & 9 & 125 \\
15B & MVA and diffuse injury & - & - & F & 24 & Right & 12 & 9 & 97 \\
16B & Fall and diffuse injury & - & - & F & 50 & Left & 12 & 9 & 104 \\
17B & Personality Disorder & - & - & M & 24 & Right & 12 & 9 & 106 \\
18B & MVA and diffuse injury & - & - & F & 62 & Left & 14 & 10 & 107 \\
19B & Fall and diffuse injury & - & - & M & 46 & Right & 20 & 10 & 95 \\
20B & Central pontine myelinosis & - & - & F & 57 & Right & 16 & 10 & 76 \\
21B & Chronic alcoholism & - & - & M & 51 & Right & 12 & 10 & 84 \\
22B & Cardiac arrest and global anoxia & - & - & M & 60 & Right & 18 & 11 & 91 \\
23B & Chronic alcoholism & - & - & M & 49 & Right & 12 & 11 \\
24B & Concussion & - & - & M & 57 & Right & 12 & 11 & 90 \\
25B & MVA and concussion & - & - & F & 54 & Right & 12 & 12 & 89 \\
26B & Nonverbal learning disability & - & - & F & 18 & Right & 12 & 12 & 92 \\
\hline
\end{tabular}

Cases 1A to 15A consist of participants with constructional apraxia in their drawings according to the Threshold Rating System. Cases 1B to 26B consist of participants with normal drawings. Lat. = laterality of lesion (left, right); $\mathrm{RT}=$ right; $\mathrm{A} / \mathrm{P}$ or Ant./Pos.= anterior or posterior lesion; Hand $=$ handedness $($ left or right); Educ $=$ years of education; MVA = motor vehicle accident; DAP Score $=$ Draw-A-Person Score [11]; pos. = posterior; inf $=$ inferior; Rating $=$ global rating of impairment on three drawing set pooled across three raters for each subject; $\mathrm{TL}=$ temporal lobe; mid $=$ middle, $\mathrm{SPL}=$ superior parietal lobule, $\mathrm{AG}=$ angular gyrus.

\subsection{Neuropsychological Assessment Battery}

In the present study, participants were assessed using the Wechsler Adult Intelligence Scale - Fourth Edition (WAIS - IV) [43], Wechsler Memory Scale - Fourth Edition [44], and the ACS Social Perception Battery [45]. These core batteries were supplemented by tests of attention, verbal and nonverbal executive function, motor and sensory functions as well as tests of personality and mood. A description of these neuropsychological tests administered is detailed elsewhere [46].

\subsection{Case Series Design}

The performance of subjects on the neuropsychological tests was determined by examining age-matched standardized scores between the drawing-normal and drawing-impaired participants. Using this method, analysis of dissociations between participants' performances on specific tests could then be carried out in the context of the patient's different lesion topographies [47]. This approach allows for a first approximation of the neural correlates of CA associated with free-drawing if undertaken in conjunction with an examination of a succession of case series. The strength of the dissociation of performances paradigm lies in its use of inferences regarding the "configuration of signs" rather than isolated test signs in pointing towards specific neuropsychological syndromes [47]. That is, more than one neuropsychological test is used in determining the neuropsychological syndrome effecting a certain patient. When brain lesions are robust enough to cause cortical neurological disorders such as CA and nonCA neuropsychological syndromes, an impaired/normal analysis of errors in performance should enable determination of some of the essential neural systems involved [39, 40].

Importantly, although the "impaired" and "normal groups" are categorized qualitatively in this way, nearly all non-CA participants with brain injuries demonstrated at least one type of neuropsychological deficit other than CA. There were very few patients in the sample of 41 patients with functional or non-ostensibly organic disorders. Further, the use of an over-arching neuropsychological theoretical orientation about the mechanisms, interactions, and functional aspects of coordinated brain systems can obviate the need for large samples when determining the neural correlates of performance of newly developed neuropsychological tests [47-49]. Participants with unambiguous constructional apraxia using the Threshold Rating System and with singular, focal, and contiguous lesions were first identified. Seven participants were identified in this manner. Six participants with lesions that were singular, focal, and contiguous without constructional apraxia were also identified.

Eight participants with constructional apraxia without lesions that were clearly able to be classified as singular, focal, and contiguous were also included to provide a better 
first approximation of the range of variance associated with CA. Finally, 20 additional control participants without CA and without lesions that were clearly discernible as singular, focal, and contiguous were included. Importantly the nonCA participants all had some form of neuropsychological disorder $(21 / 26=84 \%)$. Only five participants (two personality disorder participants, two persons with alcoholism, and one subject with developmentally-based nonverbal learning disability) did not have macro-level brain-lesion related neuropsychological disorders. Although, persons with alcoholism often do develop neurological symptoms associated with disease progression [12]. These five participants were included nonetheless to accentuate the normal range that one might find in an unselected consecutive sample of neuropsychological patients recruited in rehabilitation psychology settings.

The anterior-lesioned group consisted of participants for whom the center of mass of the lesion was anterior to the central sulcus with at least $75 \%$ of the lesion extending within the frontal lobes. The posterior-lesioned group was comprised of participants for whom the center of mass of the lesion extended within the parietal-temporal-occipital lobes. With the posterior group, $75 \%$ of the lesion was required to extend within the posterior cortices. Left and right hemisphere lesioned participants were similarly categorized as involving only one hemisphere or another. All participants' lesions were corroborated by written summaries of the neuroradiologist's reports. Visual inspection of the full neuroimaging data sets with lesion visualization software [50] in conjunction with a standard CT/MRI atlas [51] was also completed. Only participants with single, focal, and contiguous topographical lesions extent-wise were included in the case series analysis. In summary, 8 of 15 or $53 \%$ of CA participants did not have lateralized or rostral/causal focalized lesions.

There were six participants with single focal contiguous lesions who did not demonstrate CA. These non-CA participants could then be compared with one another and to the CA group to determine brain areas not likely to be involved in constructional apraxia. Hence two groups were formed. The constructional apraxia (CA) or nonconstructional apraxia (non-CA) groups, each with singular focal contiguous stroke lesions, provide the basis for a single or potentially double dissociation analyses [47]. Again, it is important to reiterate that participants with specific focal lesions were examined with dichotomous ratings on their free-drawings for the presence or absence of CA. A dichotomous rating system might facilitate the identification of the most salient features of the drawings in order to determine categorization into the $\mathrm{CA}$ or non-CA groups. Such a dichotomous rating scale might also obviate the need for drawing evaluators with detailed artistic or neuropsychological knowledge $[15,16]$. Participants with focal lesions of comparable size/severity, and who manifest other non-CA neuropsychological syndromes, yet present without CA, would suggest that a particular brain region is probably not critically involved in free-drawing related constructional apraxia. The present focus of our investigation, however, was to examine the effects of a series of single specific neuroanatomically localized lesions in the brain. In the following Results sections are the discussion of the single stroke lesion patients with and without definitive impairment in their depictions of houses, trees and persons as well as a presentation of the most salient neuropsychological aspects of each case.

\section{Results}

Figure 2 illustrates the normal drawings of three participants without constructional apraxia (CA) despite sustaining large focal single cortical contiguous lesions. These drawings were similar to normal drawings by participants without any brain lesions (not shown) and do not possess any of the abnormalities that would be associated with focal neuropsychological left or right hemispheric CA localizing signs (e.g., see Table 1 on page 62 of Ref. [29]). It is important to mention that the Threshold Rating System which was shown to be near-equivalent to the Unanimous Rating System for all of the three sets of drawings of cases 1A-7A. All seven of these patients showed significant evidence of constructional apraxia. Conversely, all the three sets of drawings of cases 1B-6B all showed significant evidence of normality with respect to drawing abilities. These three case-control participants are presented for the purpose of illustrating instances where specific locations of brain lesions, even relatively large lesions, might not cause CA. There were three additional participants with single stroke lesions without $\mathrm{CA}(1 \mathrm{~B}, 3 \mathrm{~B}, 4 \mathrm{~B})$, and these participants results will be discussed forthwith in conjunction with our model of CA - (see Figure 10).

\subsection{Non-constructionally Apraxic Drawings}

Participant 2B had an age-scaled score of 7 on the Visual Reproductions I immediate memory subtest of the WMS-IV which was within the normal range [44]. In this task, participants must remember and draw complex nonverbalizable two-dimensional line drawings after a short 10 second delay. Participant 2B's score on the Visual Reproductions II after a 30 minute delay was in the severely impaired range (age-scaled score $=1$ ). On Visual Reproductions II, participant 2B mistakenly perseverated on elements of the free-drawing task completed previously. The participant drew a house for item 1, a tree for item 2, and a person for item 3 of Visual Reproductions II. This was despite being told the WMS-IV standard instructional set. A severe impairment in episodic memory could explain the overall pattern of results. This unexpected finding could also indicate a depth of processing effect in that free-drawings are more likely to access lexical and semantic-related processes as opposed to shallow encoding of visual features only, such as contour, as in copying of the Visual Reproductions I task [52]. Participant 2B's performance on Visual Reproductions Copy was nearly flawless at 40/43 correct.

Nee et al. (2007) have shown that forgetting recently 
learned material due to interference from information learned previously or "proactive interference" involves the left ventrolateral prefrontal cortex [53]. These areas were severely damaged in participant 2B's case. The impaired delayed recall for abstract two-dimensional line drawings in Visual Reproductions II could illustrate how the lexical route can dissociate from the integral functioning of the line-by-line heuristic route (e.g., see Figure 2). The dissociations between Visual Reproductions II after a 30 minutes delay (age-scaled score $=1$ ) and participant 2B's normal house (as well as drawn figures of a tree and person not depicted above) recalled from memory are striking. These dissociations provide an excellent example of how the rich semantically imbued long-term memory associated with free-drawing (house, tree or person), and which are hypothesized to access the lexical route, might surpass via lexicosemantic priming the limited performance capacity of the slavish line-by-line copying pathway used for nonverbalizable nonsense figures. The findings of $2 \mathrm{~B}$ are perhaps not surprising since Farias and colleagues' (2006) study of aphasics provided fMRI and behavioral evidence of priming and facilitation effects on access to the naming system specifically with drawing as opposed to writing [54]. What is all the more interesting is that despite large lesions subjects 2B, 5B and $6 \mathrm{~B}$ did not demonstrate constructional apraxia - (see Figure 3 ).

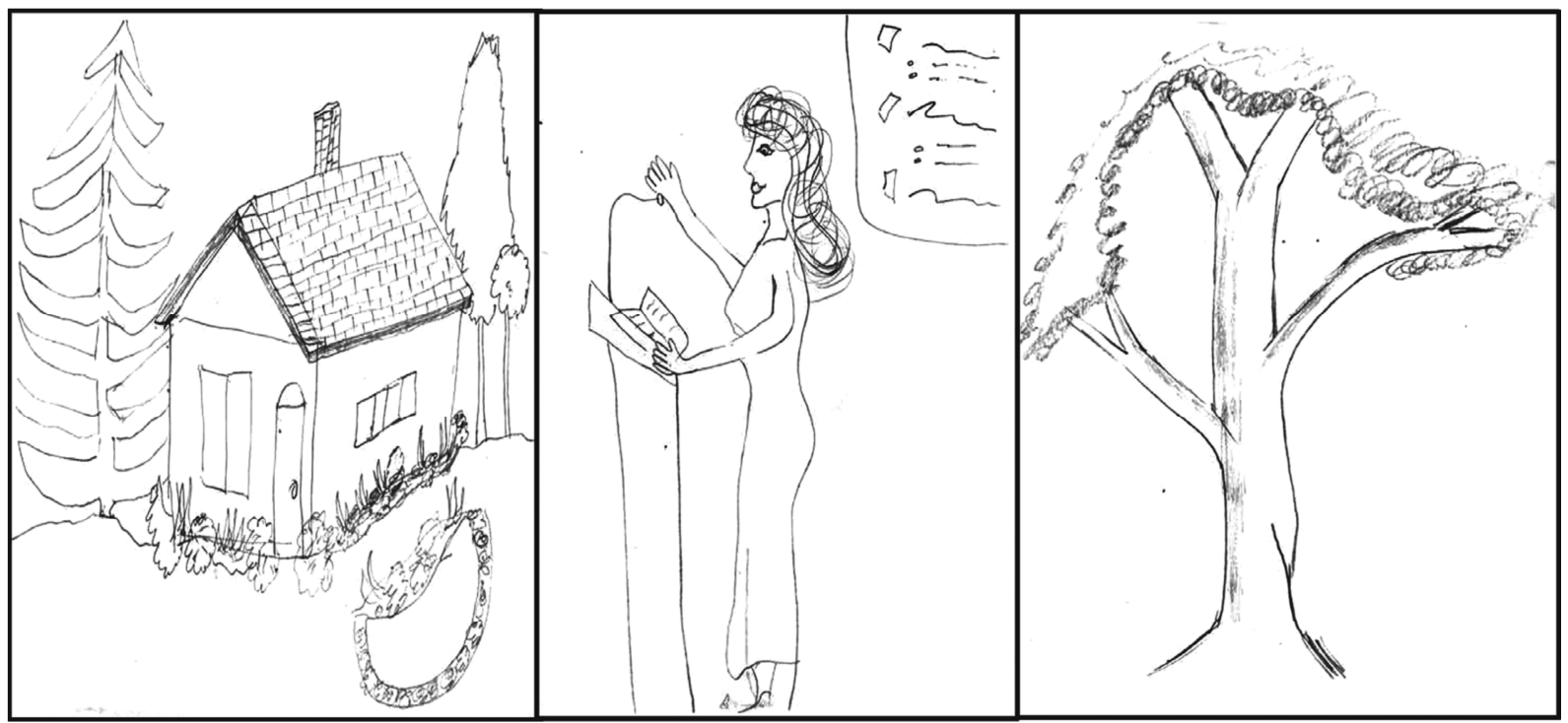

Figure 2. Examples of non-constructionally apraxic drawings. House drawing by a non-constructionally apraxic participant $2 B$ (left). Person drawing by a non-constructionally apraxic participant $5 B$ (middle). Tree drawing by non-constructionally apraxic participant $6 B$ (right).
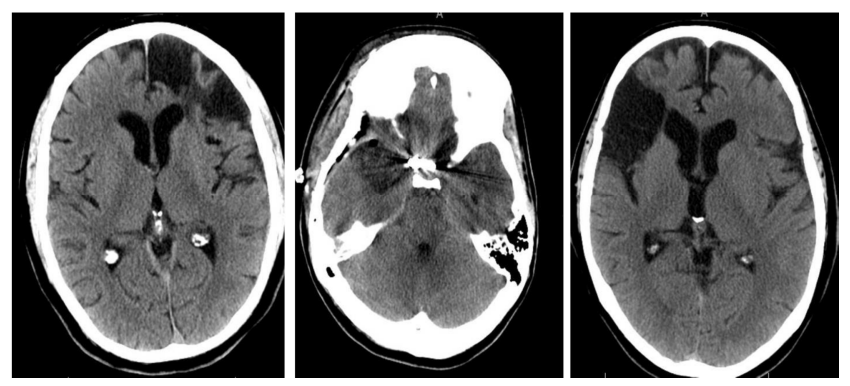

Figure 3. $C T$ scan images of participant $2 B, 5 B$ and $6 B$ 's brain lesions. Participants $2 B, 5 B$, and $6 B$ 's complete set of House, Tree, and Person drawings were rated as normal by all three raters. Participant $2 B$ 's scan depicts a wedge-shaped lesion measuring $7 \mathrm{~cm}$ in length from the left frontal pole extending to the left posterior inferior frontal lobe (left). Participant $5 B$ 's scan depicts a right anterior inferior temporal lobe lesion involving the orbitofrontal gyri (middle). Participant 6B's scan depicts a large infarct in the right posterior-inferior frontal lobe measuring $5.0 \times 4.2 \mathrm{~cm}$ and extending posteriorly to include the most rostral aspect of the right inferior temporal pole (right). According to neuroradiological convention left is right and right is left.

Participant 5B's Verbal Comprehension Index of 121 was consistent with a high level of premorbid ability. Participant 5B performed poorly on the Booklet Category Test $(\mathrm{T}=34)$ which is a test of nonverbal concept formation [55]. Participant 5B also scored poorly on the Object Decision task of the Visual Object and Space Perception Battery [56] consistent with an acquired associative agnosia which can occur after right temporal lesions [57]. Participant 6B sustained an extremely large right inferior frontal lobe lesion. The only neuropsychological deficits of note were an inability to maintain set on the Wisconsin Card Sort Test [58]. Studies have shown that loss of set errors are apt to occur in individuals with lesions encroaching into the orbitofrontal cortex of either hemisphere [59]. Participant 6B's Grooved Pegboard Test [60] left hand performance was severely impaired $(\mathrm{T}=4)$ as expected given his right inferior frontal lobe lesion [35]; although importantly this patient was right handed.

Collectively, the results show, that contrary to a significant body of prior research, frontal lesions in either hemisphere do not reliably induce $\mathrm{CA}$, at least in a free-drawing format (e.g., participants $2 \mathrm{~B}, 5 \mathrm{~B}, 6 \mathrm{~B}$ ). The pattern of results for the three aforementioned participants with specific single focal cortical lesions also imply that the most anterior reaches of the ventral stream, (mainly the left or right orbitofrontal prefrontal cortex) are not appreciably involved in free- 
drawing (e.g., 2B, 5B). The right thalamus did not appear to be involved in CA (13B) although the left thalamus and/or bilateral thalami could well be involved (14A).

\subsection{Constructionally Apraxic House Drawings}

Participant 2A's attention, visuospatial/constructional and delayed memory composite scale indices were all below the first percentile on the Repeatable Battery for the Assessment of Neuropsychological Status [61]. Immediate memory was in the normal range. Participant 2A performed at the second percentile on the Brief Test of Attention [62] and at the first percentile in the left visual field part of the Visual Search and Attention Test [63]. Performance on the right visual field of the Visual Search and Attention Test was at the $27^{\text {th }}$ percentile and within normal limits suggestive of mild left visual field neglect. However, examination of participant 2A's drawn house (shown below in Figure 4) did not reveal any apparent left visual field neglect. The lack of apparent left visual field neglect in the drawing of a house below might be an example of how the lexical and semantic encoding route depicted in purple in Figure 1 can augment damaged representational mechanisms across the divided visual field.

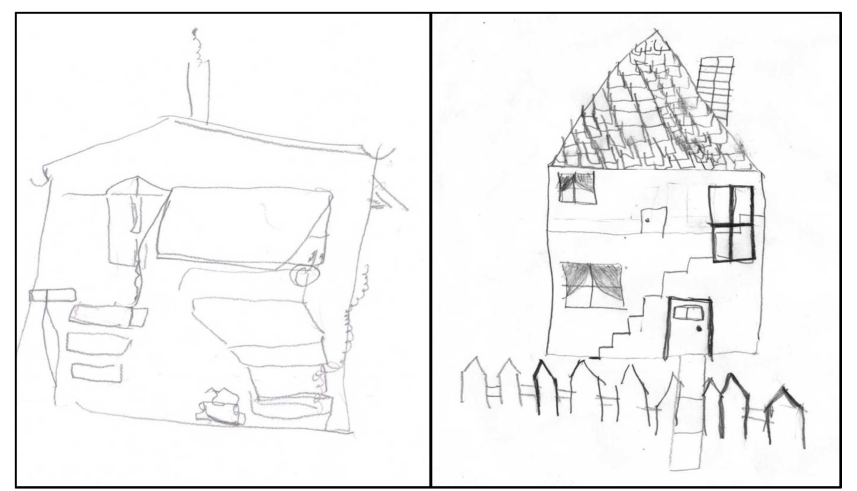

Figure 4. Constructional apraxia in house drawings of participants $2 A$ and $4 A$. House drawing by constructionally apraxic participant $2 A$. Note that the individual details are accurately represented but that there is a loss of spatial relationships (left). House drawing by constructionally apraxic participant 6 A. Note the intact global outline with the wrong orientation between the component parts (right).

Participant 4A demonstrated a discrepancy between the Verbal Working Memory Index (82) as measured by the WAIS-IV and the Visual Working Memory Index (63) as measured by the WMS-IV. This was consistent with very large right-sided parietal injury as a consequence of a brutal assault on this skilled young man. Also the Auditory Delayed Index of 95 was significantly greater than the Visual Delayed Index of 75 congruent with right-sided injury $(\mathrm{p}<0.05)$. The impairment in Visual Delayed Memory was due to low scores on Designs II (age scaled score $=4$ ) compared with Visual Reproduction II (age scaled score $=8$ ) suggesting that the manual aspect of Visual Reproductions II might be able to differentially prime performance. Participant 4A was impaired on the Ruff Figural Fluency Test $(\mathrm{T}=28)$ which is sensitive to right hemisphere lesions [64]. Participant 4A also scored in the impaired range on the Object Decision task $(\mathrm{T}=2)$ and the Silhouettes subtest $(\mathrm{T}=18)$ of the Visual Object and Space Perception Battery [56]. Both of these tasks rely on the effective encoding of the gestalt aspects of the stimulus and require the integrity of right-sided networks involved in visual classification of objects and scenes [65]. Finally, participant 4A was severely impaired in the Affect Naming $(\mathrm{T}=30)$ and Prosody-Pair Matching $(\mathrm{T}=26)$ of the Wechsler Advanced Clinical Solutions Battery [45] consistent with the right temporoparietal region's role in social and affective perception [66]. Participant 4A was previously well-adjusted socially. Participant 2A and 4A's right hemisphere lesions are depicted in Figure 5.

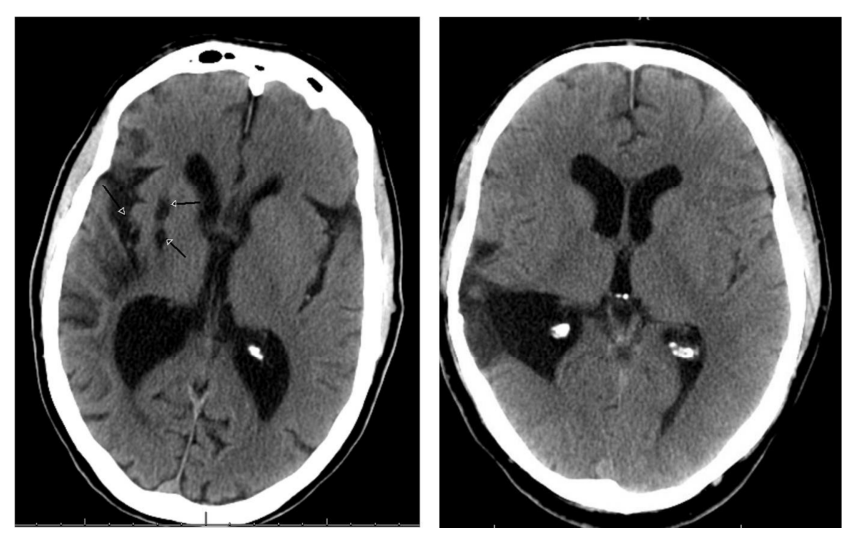

Figure 5. CT scan images of participant $2 A$ and $4 A$ 's brain lesions. Participant $2 A$ 's scan depicts a right posterior insula, right internal capsular and right caudate nucleus necrosis. See the three black arrows pointing to these features (left). Participant 4A's scan depicts a large area of gliosis present within the right posterior temporal region which extends all the way up to the right parietal lobe with large secondary dilatation of the posterior horn of the right lateral ventricle (right).

\subsection{Constructionally Apraxic Tree Drawings}

Participant 3A was significantly impaired on the Symbol Search subtest of the WAIS-IV [43] - (see Figure 6). Symbol Search involves simultaneous online monitoring and marking of one non-verbalizable design among six others and would be expected to tap the resources of a limited capacity visual buffer - (See Figure 1). Symbol Search has many of the features of a line-by-line reconstitution of the elements of a nonverbal design from one point on the page to another. The right superior parietal lobule has been shown to be essential for such visual remapping of spatial coordinates [67] and for participant 3A this brain region was severely damaged. Participant 3A's age scaled scores on Designs I and II were 9 and 4 respectively. Closer examination of Designs II revealed that loci of the problem was an inability to remember the content of the items (age scaled score $=4$ ) whereas remembrance of the spatial location of an item was intact (age scaled score $=8$ ). McCrea, Coslett and Buxbaum (2006) have shown that integrity of the dorsal occipitoparietal cortex is essential for mapping spatial locations and in 3A's case these regions were intact [68]. Damage to the right superior parietal lobule, then, could 
have resulted in a disconnection of the right dorsolateral prefrontal cortex from the right superior parietal lobule involved in spatial working memory [69].

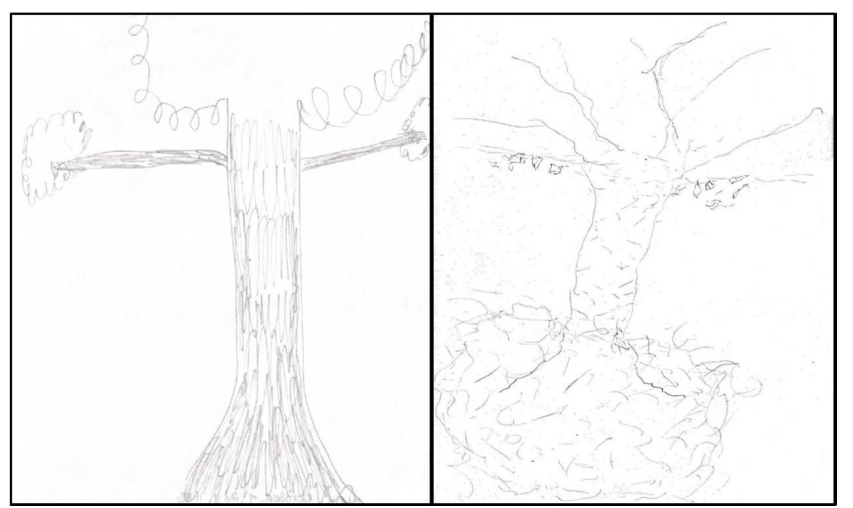

Figure 6. Constructional apraxia in tree drawings of participants $3 \mathrm{~A}$ and $6 A$. Tree drawing by constructionally apraxic participant $3 A$. Note that the individual details are accurately represented however these are in the wrong orientation to the trunk of the tree (left). Tree drawing by constructionally apraxic participant $6 A$. Note the preservation of the intact overall contour of the tree, perseveration on small details, and a vague general depiction (right).

A right superior parietal lobe lesion would also disconnect object-based mechanisms in the occipitotemporal posterior cortex with the spatial location based system through looping inferior-superior fiber tracts within the right posterior cortex and underlying white matter. In this context, although spatial location based mechanisms would be intact, these spacebased mechanisms would nonetheless be unable to manage the numerous location/object identity combinations or binding of locations with the object [68]. Participant 3A was also impaired on Part B of the Trail-Making Test $(\mathrm{T}=30)$ [70] and the Booklet Category Test $(\mathrm{T}=28)$ [55] consistent with a nonverbal executive function impairment. Prosody-Face Matching subtest of the Advanced Clinical Solutions was also impaired $(\mathrm{T}=33)$ [45] consistent with defective integration of the visual facial expressions with the melody of the human voice to understand intentions. Such integrative emotional functions are usually construed as being sensitive to the integrity of the right temporoparietal region and underlying white matter [66].

Participant 6A's standard score on the Peabody Picture Vocabulary Test - Fourth Edition of 92 was in the average range. Participant 6A was examined with the full Western Aphasia Battery - Revised [71] and on the basis of normal fluency, comprehension and poor repetition received a diagnosis of conduction aphasia. The results also suggested that participant 6A exhibited symptoms of alexia with agraphia. The alexia appeared less severe than the agraphia since reading comprehension on the WAB-R was rated at $6.5 / 10$ whereas free response writing output was listed at $0 / 10$. Moreover, spontaneous speech in oral format was rated at $9 / 10$. Both free response writing output and spontaneous speech use the same visual picture as the stimulus on the WAB-R. Lexical agraphia or dysfunction of the lexical writing system (e.g., writing irregular words to dictation:
2/10) typically involves lesions to the junction of the posterior angular gyrus and the parieto-occipital region [72]. For participant 6A, both areas were damaged. The phonological variant of agraphia (e.g., writing non-words: 0/10) has been shown by Roeltgen (2003) to involve the supramarginal gyrus [72]. For participant 6A, the posteriorsuperior aspects of the supramarginal gyrus and white matter deep to this region were also likely damaged - (see Figure 7). Hence, there were elements of both lexical and phonological agraphia which might explain the exceptionally poor score on free-response writing output (e.g., 0/10).
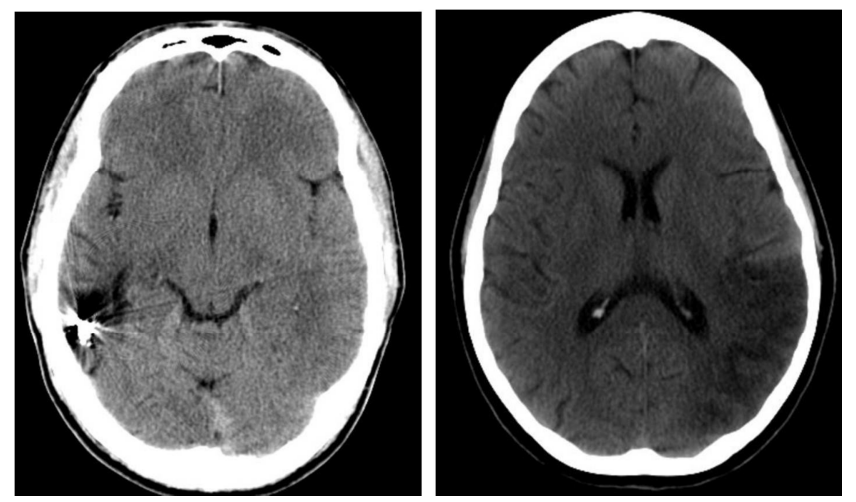

Figure 7. CT scan images of participant $3 A$ and $6 A$ 's brain lesions. Participant $3 A$ 's scan depicts atrophy in the posteriosuperior aspect of the right parietal lobe and extensive damage extending inferiorly to the right posterior middle temporal gyrus (left). Participant 6A's scan depicts an infarct extending from the posterior superior and middle temporal gyri to the occipital gyri in the left hemisphere, encompassing $9 \mathrm{~cm}$ in the axial plane. Superiorly, the left angular gyrus and left supramarginal gyrus were also damaged (right).

Importantly, general praxis was near normal as evaluated by the apraxia subtest of the Western Aphasia Battery Revised (52/60). However, there did appear to be deficits in pantomime recognition on the WAB-R. Pantomime production was especially poor on three of five ceiling-level difficulty items. These items provide imitative cues demonstrated by the examiner and the examinee is then required to perform the assigned gesture. Pantomime recognition failure is often associated with impaired reading comprehension and various associated alexias. Lesions to the left angular or supramarginal gyri are apt to cause pantomime recognition deficits [73]. Participant 6A performed poorly on the Silhouettes subtest of the Visual Object and Space Perception Battery $(\mathrm{T}=25)$. The Silhouettes subtest uses three-dimensional shadow images in which participants are required to name animals or inanimate common objects in unusual views. Participant 6A's raw score on Silhouettes of $13 / 20$ was substantially below that of left hemisphere lesioned participants tested in the standardization sample. This low score could be illustrative of the extensive damage to the occipitotemporal cortex within the left hemisphere in Participant 6A. The base rates for such a low score on Silhouettes would occur with a frequency of less than $2 \%$ of the standardization sample.

Participant 6A used many circumlocutions to describe the objects (e.g., rhinoceros = they have a horn on their nose 
and they go in water). Participant $6 \mathrm{~A}$ was able to identify by instrumental actions three items from the common objects list (e.g., wrench $=$ "where the examinee gestured tool usage"). Participant 6A similarly scored poorly on Progressive Silhouettes $(\mathrm{T}=20)$ in which two objects are rotated in incremental degrees from the lateral view of 90 degrees so that critical distinctive features are no longer visible. Participant 6A was unable to name some of these common objects in the lateral view but could gesture their use as with the Silhouettes subtest suggetive of a potential diagnosis of optic aphasia. Optic aphasia characterized by a deficit in naming objects presented visually, usually as a result of left occipitotemporal lesions; although patients with this disorder can name objects to tactile palpation or to verbal description [74].

\subsection{Constructionally Apraxic Person Drawings}

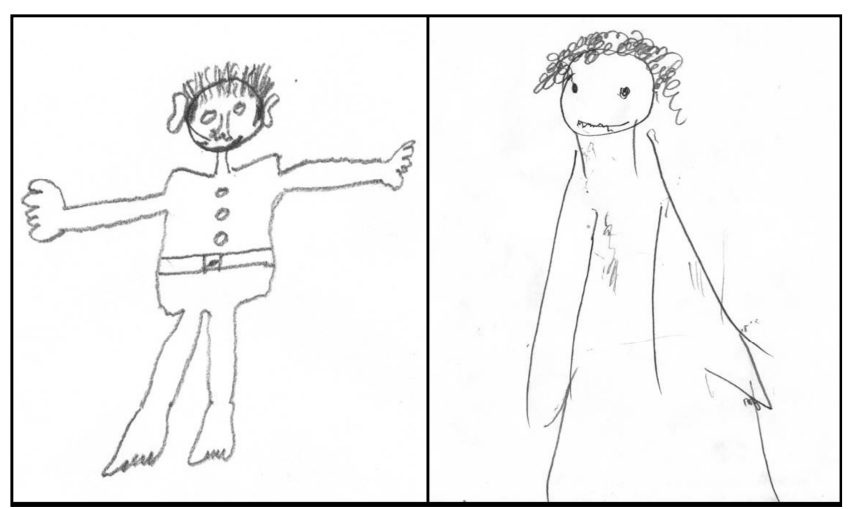

Figure 8. Constructional apraxia in person drawings of participants $1 A$ and 7A. Person drawing by constructionally apraxic participant $1 A$. Note the loss of accurate spatial relationships between appendages, head, and torso (left). Person drawing by constructionally apraxic participant $7 A$. Note intact contour outline of the body, with poor angle representation between limbs and vague overall appearance (right).

Participant 1A scored in the very low range on Delayed Memory of the RBANS [61] - (see Figure 8). The impairment in delayed memory was particularly prevalent in the nonverbal domain consistent with the right lateralized lesion - (nonverbal recall $40 \%$ correct versus verbal recall $71 \%$ correct). Participant $1 \mathrm{~A}$ demonstrated a difficulties with Finger Tapping, Grip Strength [75] or Grooved Pegboard with the left hand; although importantly he was able to draw with his dominant right hand. Left hemiplegia is often associated with pronounced anosognosia which Vocat, Staub, Stroppini and Vuilleumier (2010) have shown is apt to occur in cases with extensive damage to the right insula [76]. Participant 1A demonstrated pronounced anosognosia and right insular damage.

Participant 7A suffered a left temporoparietal stroke with an apparent constructionally apraxic drawn person - (see Figure 8). From an ipsative analysis, participant 7A had relative strengths on Figure Weights (age scaled score $=6$ ) and Block Design (age scaled score $=5$ ) on the WAIS-IV [43]. Participant 7A had relative weaknesses on Similarities (age scaled score $=1$ ) and Vocabulary (age scaled score $=2$ ).
Participant 7A's Visual Reproductions I and II age scaled scores on the WMS-IV were 1 and 4, respectively [44]. Visual Reproductions Copy age scaled score was in the very low range at 4. However, participant 7A's Visual Reproductions Recognition was in the normal range (age scaled score $=6$ ), implying that the five subtest items had initially been encoded correctly. None of the Visual Reproductions items are easily verbalizable and would thus have been expected to use the line-by-line heuristic route (see Figure 1). Given the extensive left hemisphere damage, the rate-limiting step in processing might be expected to be the visual buffer. In the case of participant $7 \mathrm{~A}$ components of the visual buffer BA 40 (supramarginal gyrus) and 7 (superior parietal lobule) were damaged within the left hemisphere $9-$ (see Figure 9).
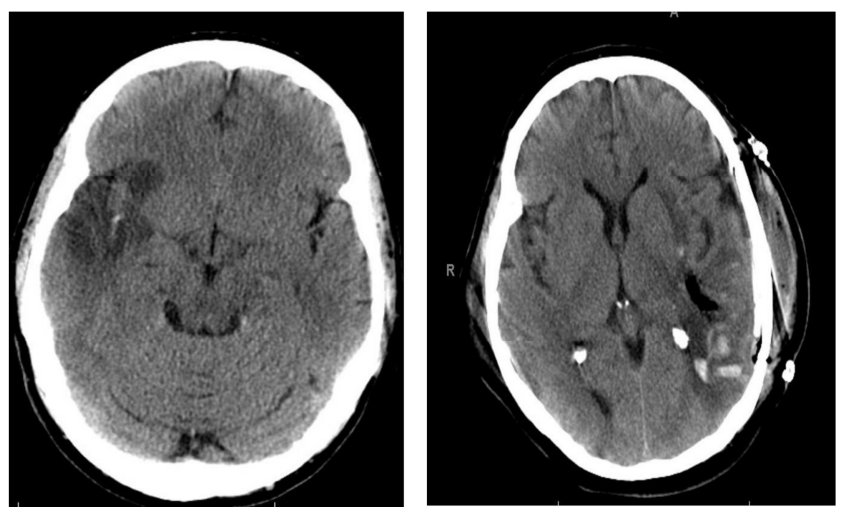

Figure 9. CT scan images of participant $1 A$ and $7 A$ 's brain lesions. Participant $1 A$ 's scan depicts a right anterior temporal lobe, right insula, and right basal ganglia stroke (left). Participant 7A's scan depicts a left temporoparietal stroke with damage to the left angular and left supramarginal gyrus. There was also damage to the left inferior, middle, and superior temporal lobes in addition to damage to the left superior parietal lobule (right).

Participant 7A completed the Multilingual Aphasia Examination [77] at 10 months after the stroke. Participant 7A was found to be impaired on repetition and fluency and had intact comprehension consistent with a diagnosis of Broca's aphasia. Persistent Broca's aphasia is apt to occur after large lesions, including not only Broca's area proper, but also areas surrounding the Rolandic fissure. Moreover the insula, anterior parietal lobe and subcortical regions deep to these areas are often damaged in persistent Broca's aphasia [78]. In participant 7A's instance, there was damage to the insula and deep white matter underlying these areas. Performance on the Color Trails was also impaired $(\mathrm{T}=20)$ suggestive of an impairment in mental flexibility [79].

The last constructionally apraxic participant to be evaluated is participant 5A -- (drawings and lesions are not shown). Participant 5A suffered a large right temporoparietal stroke. The lesion extended from the right inferior temporal gyrus to the right medial temporal lobe and all the way to the medial temporal gyrus. There was unanimity in the agreement of damage to both the house and tree drawings, and participant 5A had the second highest average impairment rating (See Table 3). Participant 
5A's Verbal Comprehension Index of 108 was 41 standard score points higher than the Perceptual Organization Index of 67 which would occur with a base rate of less than $1 \%$ in the standardization sample of the WAIS-III [80]. This pattern of results was consistent with the right lateralized nature of the injury. Participant 5A's age scaled score on Block Design was 4 and on Visual Reproductions I of the Wechsler Memory Scale - III (WMS-III) it was 5, again consistent with CA [81].

Participant 5A demonstrated a significant difference in favor of Auditory Immediate Memory (97) compared with Visual Immediate Memory (68) consistent with the locus of the lesion. Participant 5A's Visual Reproduction II Delay on the WMS-III was within the normal range implying that successful encoding of the nonverbal figure had originally taken place (age scaled score $=7$ ). Participant 5A's performance on Visual Reproduction II - Copy was equal to an age scaled score of 2, and Visual Reproductions Recognition score was 6 . Thus, these results imply that nonverbalizable two-dimensional figures were initially encoded correctly but that visually-guided copying was impaired, perhaps via damage to the visual buffer. For participant 5A, there was evidence of a tendency to orient drawings diagonally on the page which has been shown to be associated with right hemisphere lesions and CA (See Table 1 on pg. 62 of Ref. [29]).

For participant 5A, the Spatial Span subtest of the WMSIII was impaired (age scaled score $=4$ ), which is consistent with sustaining a lesion extending within the right temporal lobe and concomitant poor nonverbal sequencing [82]. Collectively these results imply some degree of damage to the line-by-line heuristic route, and examination of the CT scans revealed damage to the right superior parietal lobe in the right hemisphere. The nature of the lesions would suggest damage to both the spatial working memory systems supporting maintenance as well as direct damage to the visual buffer [83]. Auditory attention measured by the Brief Test of Attention [62] was severely impaired ( $\mathrm{T}=29)$, as was visual attention $(\mathrm{T}=29)$ as measured by the Visual Search and Attention Test [63]. These dual visual and auditory attention deficits are consistent with multimodal attention functions subserved by the damaged right parietal lesion [84].

Although left visual neglect was directly observed on the Visual Search and Attention Test, there was no evidence of neglect in any of participant 5A's drawings, suggesting that the lexical route might be augmenting representational elaboration processes associated with each drawing. Future work might examine the relationship between semantic elaboration of drawings and neglect in nameable verbal and abstract designs. The Tower of $\operatorname{London}^{\mathrm{DX}}[\mathrm{T}=26]$ was impaired [85] as was the Ruff Figural Fluency Test [T=28], consistent with the right lateralized lesion [64] and a concomitant nonverbal executive function impairment. Participant 5A performed in the impaired range $(\mathrm{T}=29)$ on the Booklet Category Test and the number of categories achieved on the Wisconsin Card Sort Test $(\mathrm{T}<20)$ suggesting difficulties in concept formation and mental flexibility, respectively.

\section{Discussion}

Figure 10 implicates the involvement of the left temporoparietal region (7A) in constructing person representations. Brain lesion and functional neuroimaging studies have previously shown that the left inferior parietal lobe is implicated in autotopagnosia [86] or the loss of spatial knowledge about one's own body. However, construction of person representations can also occur in participants with reversed autotopagnosia and right temporoparietal lesions [87]. Two female participants were found to have lesions within the vicinity of this brain region without CA (3B, 4B), one of whom was left-hand dominant. Left-handers often have some degree of higher base rates of bilateral representation of language [39] and perhaps other non-linguistic spatial functions. This might explain the nonsignificance of participant 3B and 4B's lesions on CA. However the precise relationship between $\mathrm{CA}$ associated with person drawings and an inability to point to body parts on command in autotopagnosia is beyond the scope of this study.

Subject 6A's lesion was localized to the left posterior temporal lobe and it extended into the occipital gyri. Damage included the posterior supramarginal gyrus and white matter deep to this structure. Such lesions can result in deafferentiation of inferior occipitotemporal located visual word form areas from temporoparietal receptive language areas. Not surprisingly, participant 6A demonstrated alexia with phonological agraphia. The phonological form of agraphia has been shown by Roeltgen (2003) to almost invariably involve damage to the supramarginal gyrus. Participant 6A scored 37th out of 37 participants on standardized WRAT-4 [88] Spelling scores adjusted for premorbid IQ $(\mathrm{z}=3.18, \mathrm{p}<0.001)$. The only other patient with such poor Spelling scores in this range was subject 7A with a left temporoparietal lesion also extending all the way to the left supramarginal gyrus. Not surprisingly, participant 7A scored 36 out of 37 on standardized WRAT-4 Spelling scores adjusted for premorbid IQ $(\mathrm{z}=2.22, \mathrm{p}=$ 0.02). Shim and colleagues (2012) have shown that damage to the left supramarginal gyrus or inferior frontal gyrus was negatively correlated with proficiency on non-word spelling in primary progressive aphasics [89]. Non-word spelling is a primary method of evaluating the integrity of phonological decoding.

Participant 10A, who had a right superior cerebellar lesion, was also marginally impaired on Spelling $(\mathrm{z}=$ 0.90 ) and scored 33 out of 37 on this test. Right superior cerebellar regions are routinely activated during fMRI tasks involved in subvocal articulation and other linguistic processes [69]. These subvocal articulatory loops presumably are instantiated by frontal-ponto-cerebellar decussated tracts and are involved in verbal working memory processes [90]. Unfortunately, participant $10 \mathrm{~A}$ 
was not administered tests of verbal working memory. However, the empirical connection between verbal working memory capacity and the integrity of the right superior cerebellar lobe is robust. Finally, in the left hemisphere, large lesions of either the superior frontal lobe (1B) or the inferior frontal lobe (2B) did not result in $\mathrm{CA}$, which is consistent with the perspective that CA, inso-far as free-drawing is concerned, is highly dependent upon the integrity of posterior cortical networks. Collectively, analysis of results of participants with left hemisphere lesions support a linguistically-mediated role for performance on free-drawing tasks such as the housetree-person $(6 \mathrm{~A}, 7 \mathrm{~A}, 10 \mathrm{~A})$, as well as a unique role for the lexical route to word access in these processes.

Participants $6 \mathrm{~A}$ and $7 \mathrm{~A}$ were impaired on the tree and person drawings, respectively. A bilateral thalamic infarct found in patient (14A) resulted in impairment in the person drawing only, whereas the unilateral lesioned right thalamic lesioned patient (13B) was not found to be impaired in any drawings. A review of 465 participants with vascular thalamic lesions demonstrated that two-thirds of bilateral thalamic participants presented with CA [91]. A recent study found CA in a rare right hander with crossed aphasia. This crossed aphasia patient's CA emerged after a right thalamic lesion and thus there was the strong presumption of right hemisphere dominance for language functions [92]. In view of positive and negative indices of $\mathrm{CA}$ in participant $14 \mathrm{~A}$ and $13 \mathrm{~B}$, respectively, the results speak to a potential left thalamic role in 14A's defective person drawings. The left ventral posterior-lateral nuclei of the thalamus encodes detailed somatotopic representations of the body for the language dominant left hemisphere, and might play an essential role in sensorimotor mapping in CA [93]. These results could speak to a role for representations of the body schema's involvement in constructional apraxia for some classes of stimuli such as full human bodies and body parts.

There was more unanimity in regards to the pattern of results for intra-hemispheric lesions within the right hemisphere and these results were robust. These findings may reflect the more distributed language system in the left hemisphere and its hypothesized interaction with CA's motor praxic mechanisms involvement in free-drawing. Nonetheless, participants $3 \mathrm{~A}, 4 \mathrm{~A}$ and $5 \mathrm{~A}$ had large right temporoparietal lesions and were impaired on the tree (3A), or house and tree $(4 \mathrm{~A}, 5 \mathrm{~A})$, respectively. None of these individuals $(3 \mathrm{~A}, 4 \mathrm{~A}$ or $5 \mathrm{~A})$ were impaired on the person drawings. The drawing results of $3 \mathrm{~A}, 4 \mathrm{~A}$ and $5 \mathrm{~A}$ could speak to the lack of involvement of the right temporoparietal cortex in person drawings. Indeed there is a large literature suggesting that person representations have a dedicated neural architectural representation in the brain which is different from other types of living and non-living objects or entities [94].

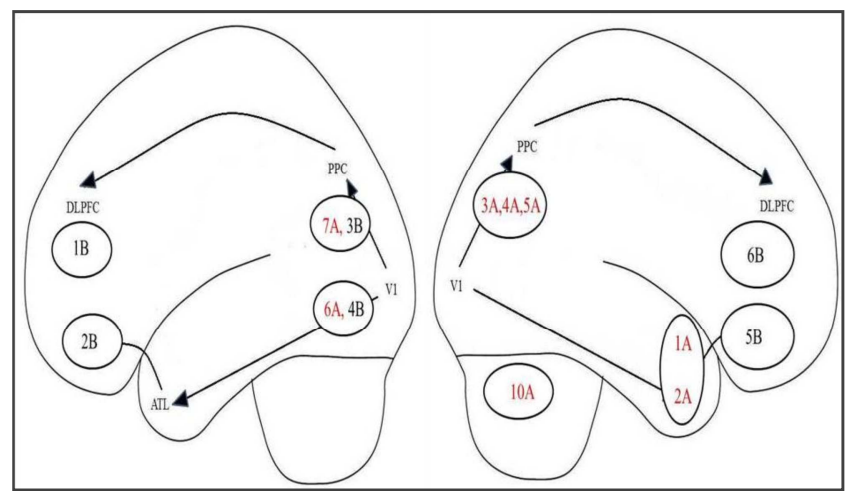

Figure 10. Topography of brain lesion's effects on constructional apraxia conceptualized in terms of Milner and Goodale's (2006) theory of ventral and dorsal streams [110]. The left image depicts the left distribution of lesions in the cortex whereas the right image depicts the distribution of lesions in the right hemisphere. Legend: $V 1=$ primary visual cortex, $P P C=$ posterior parietal cortex, $A T L=$ anterior temporal lobe, DLPFC = dorsolateral prefrontal cortex. Note that lesions $2 B$ and $5 B$ are localized within the orbital gyri in the left and right hemispheres, respectively. $1 A$ and $2 A$ are depicted as an ovals to illustrate the medial extension of these lesions to encompass the insula. The red letter-number combinations indicate that lesions resulted in constructional apraxia. The black letter-number combinations indicate that lesions did not result in constructional apraxia. Only participants with singular focal contiguous lesions were included in this summary analysis.

Participant 1A, who had damage to the right anterior temporal lobe, right insula and right basal ganglia was impaired on the person drawing only. Participant 2A, who had damage to the right basal ganglia and insula, was impaired on all three drawings. The commonality of impairment on the person drawings of participants $1 \mathrm{~A}$ and 2A may fit with Karnath and Bayer's (2010) theory that the right insula is pivotally involved in perceptions of limb ownership, as well as self-perceived limb movement and the body schema [95]. These authors noted that right insular lesions were often associated with anosognosia or unawareness of deficits. Indeed, participants $1 \mathrm{~A}$ and $2 \mathrm{~A}$ suffered anosognosia or unawareness of deficit. Finally, participants with right superior anterior prefrontal cortex (6B) and right inferior prefrontal cortex (5B) lesions did not reliably produce free-drawing-related CA.

Smith (2009) noted that drawing tasks, which are practical to administer in hospital settings, have been shown to be sensitive to several functional deficits [96]. Drawing tasks differ from other neuropsychological tasks in (i) being not underpinned by a rich understanding of drawing processes, and (ii) interpretation of these tasks has been hampered by predominantly qualitative mode of inquiry and a lack of instantiation in neuropsychological underlying neural correlates of functioning. Smith noted that drawing from memory has been essential in understanding visual imagery deficits. Riddoch's study of patient DW with a left temporoparietal lesion, found that DW had good copying skills with an inability to draw objects to command, suggestive of defective image generation processes [97]. Patient 7A with a left temporoparietal stroke lesion similarly had difficulties drawing a person to verbal prompting and 
scored 23 out of 27 participants on the Visual Puzzles subtest (age scaled score $=4$ ).

Smith (2009) [96] also noted that Grossi and colleagues' (1986) description of patient AP with a left occipital lesion who was unable to draw from memory [98]. Our patient 6A, also with a left occipitotemporal lesion had difficulties drawing a tree from memory and scored 27 out of 27 participants on the Visual Puzzles subtest (age-scaled score $=$ 2). Based on research with similar types of tasks, the Visual Puzzles subtest of the WAIS-IV likely relies on the left temporoparietal junction for image generation processes [46]. In Figure 10, the left temporoparietal junction would be uniquely situated to link the visual buffer with the anterior fusiform or occipitotemporal areas.

One of the most important findings of this study was that lexical and semantic processes appeared to be essential to facilitate access to long-term visual memory and subsequent building of complex representations of real world objects such as a houses, trees, or persons. There were significant differences in performance between $\mathrm{CA}$ and non-CA participants on the Spelling subtest of the WRAT-4 [88] suggestive of damage to phonological processing mechanisms. Efficacy of phonological processing associated with spelling may index the intact nature of key visual perceptual pathways (such as objection recognition systems) to visual imagery and visual semantics [65]. Trojano and Grossi (1994) noted that left posterior lesions were apt to cause deficits in visual imagery in addition to phonological decoding difficulties [99]. Booth and colleagues found left inferotemporal activation in an fMRI study when participants performed a spelling task on auditory presented words $[100,101]$.

Cohen et al. (2004) found that the visual word form area in the left occipitotemporal cortex was strictly visual and unimodal in nature whereas a lateral inferotemporal multimodal area appeared to function as a convergence zone linking orthography, phonology and semantic information [102]. This supramodal word processing area was located laterally and anterior to the visual word form area and was activated by either spoken or written words irrespective of modality. Comprehension shares the property of a dependence upon semantic memory retrieval and access to the memory of words which is dependent upon the integrity of the left middle temporal gyrus and left inferior frontal sulcus [103]. These regions are connected by the arcuate fasciculus in the phonological reading route extending posteriorly and inferiorly from the occipitotemporal sulcus's visual word form area to the supramarginal gyrus and on towards the inferior opercular regions of Broca's area [104].

A surprising finding was the lack of effect of superioranterior frontal lobe lesions in either hemisphere causing CA. There have been isolated reports of participants with frontal variant of frontotemporal dementia that develop newfound talents in drawing of realistic objects [105]. Although these reports may have been sensationalized in the popular media Miller and colleagues have suggested that the appearance of such talents in the beginning stages of dementia could be a function of disinhibitory effects of frontotemporal disease processes on posterior cortical perceptual-motor modules. These findings have been frequent enough to be noticed by dementia researchers and suggest that, if anything, the frontal cortex may not be required for the composition of freedrawings that are heavily reliant on lexical and semantic knowledge. The results do perhaps point towards the essential role of the temporal, parietal and occipital lobes in free-drawing.

According to Figure 10 the premotor cortex would likely be involved in contingent planning and action programming. The intact caudal dorsolateral prefrontal cortex and anterior cingulate would be involved in inspection processes associated with both the lexical route and the line-by-line heuristic. Schwartz et al. (2011) noted that the anterior temporal lobe codes for taxonomy or classes of objects (e.g., apples and oranges) [106]. In contrast, this large lesion mapping study found that the left temporoparietal junction codes for thematic or function knowledge such as between a "bird and a nest." There is more recent work showing how these posterior percept building modules connect with more anterior cognitive and motor control processes. Mandonnet et al (2000) showed that the left inferior longitudinal fasciculus connects the posterior occipitotemporal regions to the temporal pole [107]. Their study showed that electrocortical stimulation of the posterior-inferior arcuate fasciculus could result in phonemic paraphasias illustrating the putative role of this region in phonological recoding. In Figure 10 the semantic system would include Brodmann's areas 38 bilaterally or the temporopolar areas, in addition to Brodmann's areas 20, 21 within the left hemisphere.

The longitudinal fasciculus traverses forward and downwards from the primary visual cortex where it contacts the left occipitotemporal region involved in visual perception and visual imagery, as well as encoding of phonological representations. The left longitudinal fasciculus would then traverse towards the left temporopolar region [108]. Within the temporopolar regions high level verbal object representations could be accessed through facilitation of the lexical and semantic systems. At the most inferior and posterior reaches of the left arcuate fasciculus these phonological representations traverse upwards towards the visual buffer and posterior parietal cortex. Callosal fibers linking the left and right superior parietal and supramarginal gyri through the posterior half of the callosum could then begin to build representations of a drawn schema. The right superior parietal and supramarginal gyri might play a dominant role in developing the global outline of the schema whereas the left homologues of these areas would fill in the details. Furthermore, the right frontoparietal dorsal stream pathway would function as a spatial working memory sketchpad during this iterative process. The ventral orbitofrontal cortices in do not appear to be involved in this model of free-drawing from memory.

\section{Conclusion}

Extensive compilations of a cases with single focal 
unilateral contiguous lesions throughout the brain should enable a further elaboration of, or modification(s) to, this model of constructional apraxia. Such a model would naturally involve metrical interactions of the object recognition, visual memory, visual imagery, visuospatial attention, lexical, semantic and motor systems involved in free-drawing. It does not appear that conventional neuropsychological drawing tasks such as the clock drawing task will capture the essence free-drawing (e.g., see Ref. [109]) because these tasks do not have many meaningful lexiosemantic associates. Other interesting concepts to study in future free-drawing research would be drawing under conditions of blindsight and with minimal prompting or under conditions of sensory deprivation. Pharmacological studies of both a practical (e.g., clinical trials of medications) and theoretical (e.g., experimental neurological research) nature could also be begun. Future studies will likely benefit from a compilation of (i) advanced neuropsychological models of free-drawing, (ii) sophisticated tools with which to analyze free-drawings components (e.g., fMRI compatible drawing tablets and standardized drawing psychometric instruments with rich semantic associates), and (iii) network interaction models of free-drawing using statistical techniques such as structural equation modeling.

It is acknowledged that this model of the functional neuroanatomy associated with free-drawing related constructional apraxia will likely require future elaborations and direct tests of hypotheses deduced from it. At present it is overly simplistic and does not do justice to contemporary models of parallel distributed processing. However it is a beginning for research to start upon as it is based on all widely accepted previously published models. Arguably many of the most substantial components of the model have been outlined (Figure 1). A current problem is that neuropsychological tests reputed to identify constructional apraxia could potentially lack ecological validity. As an example, in practical everyday life, drawings are of central importance in schematics for the visual arts, architecture, blueprint reading and the recreational doodlings of amateur and professional artists. Free-drawing related constructional apraxia is likely involved in functional disability for many acquired brain injury patients. While it is understood that there are potential limitations of this modest sample of focal lesion patients in understanding constructional apraxia large consecutive case series with imaging, broad-spectrm neuropsychological asessments in the context of plausible theoretical models is the proper approach to take for future investigators. Judged by the paucity of literature on freedrawing-related constructional apraxia, such a detailed case-by-case analysis and subsequent group average (cognitive neuroscience approach) should prove to be a fruitful and productive endeavor.

\section{Acknowledgements}

This research was conducted while Simon McCrea, Ph.D. was a registered doctoral psychologist (provisional) at the Adult Rehabilitation Program of the Wascana Rehabilitation Centre in Regina, Saskatchewan, Canada. This research was granted ethical consent to proceed by the Research and Performance Support institutional review committee of the Regina Qu'Appelle Health Region at the Wascana Rehabilitation Centre in Regina, Canada in the summer of 2010. This research was completed in the summer of 2012. All research participants provided their informed written consent in accordance with the Declaration of Helsinki [32]. This study was conducted in accordance with the principles of the Canadian Tri-Council Policy Statement: Ethical Conduct for Research Involving Humans [31]. We thank the research participants for their involvement with our study. The author of this study reports no conflict of interest.

\section{Appendix}

Instructions for Drawing a Person.

"I want you to draw a picture of yourself. Be sure to draw your whole body, not just your head, and draw how you look from the front, not from the side. Do not draw a cartoon or stick figure. Draw the very best picture of yourself that you can. Take your time and work carefully." Verbatim instructions from Reynolds \& Hickman, 2004 manual [10].

Instructions for Drawing a House: Adapted instructional set [10].

"I would like you to do some more drawings. I want you to draw a picture of a House. Be sure to draw the whole House. Do not draw a cartoon or stick figure. Draw the very best picture of a House that you can. Take your time and work carefully. Go ahead."

Instructions for Drawing a Tree: Adapted instructional set [10].

"I would like you to draw one more drawing. I want you to draw a picture of a Tree. Be sure to draw the whole Tree. Do not draw a cartoon or stick figure. Draw the very best picture of a Tree that you can. Take your time and work carefully. Go ahead."

\section{References}

[1] J. Clottes, Return to the Chauvet Cave: Excavating the Birthplace of Art, London: Thames and Hudson, 2003.

[2] A. Chatterjee, The Aesthetic Brain: How We Evolved to Desire Beauty and Enjoy Art, New York: Oxford University Press, 2013.

[3] M. Crotty, The Foundations of Social Research: Meaning and Perspective in the Research Process, Washington, DC: Sage, 1998.

[4] M. F. Schwartz and G. S. Dell, "Case series investigations in cognitive neuropsychology,” Cogn. Neuropsychology, vol. 27, pp. 477-94, 2010.

[5] R. K. Yin, Case Study Research: Design and Methods Second Edition, London: Sage, 1994. 
[6] F. Goodenough, Measurement of Intelligence by Drawings, New York: World Books, 1926.

[7] S. Kahill, "Human figure drawings in adults: An update of the empirical evidence 1967-1982," Can. Psychol., vol. 25, pp. 269-92, 1984

[8] C. H. Swenson, "Empirical evaluation of human figure drawings,” Psychol. Bull. vol. 54, pp. 431-66, 1957.

[9] C. H. Swenson, "Empirical evaluation of human figure drawings 1957-1966," Psychol. Bull. vol. 70, pp. 20-44, 1968.

[10] J. A. Naglieri, Draw-A-Person: A Quantitative Scoring System. San Antonio, TX: Psychological Corporation, 1998.

[11] C. R. Reynolds and J. A. Hickman, J. A. DAP: IQ: Draw-APerson Intellectual Ability Test for Children, Adolescents and Adults, Austin, TX: ProEd, 2004

[12] American Psychiatric Association. Diagnostic and Statistical Manual of Mental Disorders: Fifth Edition, Arlington, VA: American Psychiatric Association, 2013.

[13] K. M. Heilman and E. Valenstein, Clinical Neuropsychology Fifth Edition. New York: Oxford University Press, 2012.

[14] D. S. Tulsky, D. H. Saklofske and G. J. Chelune et al. (Eds.) Clinical Interpretation of the WAIS-III and the WMS-III, New York: Academic Press, 2003.

[15] D. Ogdon, Psychological Signs, Symptoms and Syndromes. Los Angeles: Western Psychological Services, 2001.

[16] D. Ogdon, Psychodiagnostics and Personality Assessment: A Handbook - Third Edition. Los Angeles: Western Psychological Services, 2004.

[17] R.W. Belter and C. Piotrowski, "Current status of doctorallevel training in psychological testing," J. Clin. Psychol. vol. 57, pp. 717-726, 2001.

[18] L. Bender, Instructions for the Use of the Visual Motor Gestalt Test, New York: American Orthopsychiatric Association, 1946.

[19] H. A. Murray, Thematic Apperception Test - Manual. Cambridge, MA: Harvard University Press, 1943.

[20] W. J. Camara, J. S. Nathan and A. E. Puente, "Psychological test usage: Implications in professional psychology," Prof. Psychol-Res. Pr. vol. 31, pp. 141-54, 2000.

[21] S. J. Lally, "Should human figure drawings be admitted in court?” J. Pers. Assess. vol. 76, pp. 135-9, 2001.

[22] L. Bender, "The drawing of a man in chronic encephalitis in children,” J. Nerv. Ment. Dis. vol. 41, pp. 277-86, 1940.

[23] S. Jordan, "Projective drawings in a cerebellar disorder due to chicken pox encephalitis," J. Proj. Tech. Pers. Assess. vol. 34, pp. 256-58, 1970.

[24] R. Koida, R. and K. Fujihara, "A study on HTP organic signs,” Jpn. J. Psychol. vol. 63, pp. 277-80, 1992.

[25] B. C. Meyer, F. Brown and A. Levine, "Observations on the House-Tree-Person drawing test before and after surgery," Psychosomatic Medicine. vol. 17, pp. 428-454, 1955.

[26] H. Michal-Smith, "The identification of pathological cerebral function through the H-T-P technique," J. Clin. Psychol. vol. 9, pp. 293-95, 1953.
[27] M. Rezikoff, and D. Tomblen, "The use of human figure drawings in the diagnosis of organic pathology," J. Consult. Psychol. vol. 20, pp. 467-470, 1956.

[28] M. Riklan, T. Zahn and L. Diller, "Human figure drawings before and after chemosurgery of the basal ganglia in Parkinsonism,” J. Nerv. Ment. Dis. vol. 135, pp. 500-506, 1962.

[29] S. McCrea, "A neuropsychological model of free-drawing from memory in constructional apraxia: A theoretical review," Am. J. Psychiat. Neurosci. vol. 2, pp. 60-75, 2014. doi: 10.116481/j.ajpn.20140205.11.

[30] S. McCrea and D. Chomiak, "Drawing-from-memory in constructional apraxia: Effects of focal cortical lesions on performance," J. Psychol. Psychother. Res. 2: 2-17, 2015. doi: http://dx.doi.org/10.12974/2313-1047.2015.02.01.1.

[31] Canadian Institute of Health Research, Natural Sciences and Engineering Research Council of Canada, and Social Sciences and Humanities Research Council of Canada, Tri-Council Policy Statement: Ethical Conduct for Research Involving Humans, December 2010.

[32] Declaration of Helsinki, Ethical Principles for Medical Research Involving Human Subjects - Seventh Revision. World Medical Association, Authors, 2013.

[33] C. M. Braun, I. Montour-Proulx, S. Daigneault, et al. "Prevalence, and intellectual outcome of unilateral focal cortical brain damage as a function of age, sex and aetiology," Behav. Neurol. vol. 13, pp. 105-116, 2001.

[34] J. Roquer, A. R. Campello and M. Gomis, "Sex differences in first-ever acute stroke,” Stroke, vol. 34, pp. 1581-85, 2003.

[35] M. D. Lezak, Neuropsychological Assessment - Fourth Edition. New York: Oxford University Press, 2004.

[36] C. Hardyck and L.F. Petronovich, Left-handedness. Psychol. Bull. vol. 84, pp. 385-404, 1977.

[37] NCS Pearson, Test of Premorbid Functioning: Advanced Clinical Solutions for Use with WAIS-IV and WMS-IV. San Antonio, TX: Pearson Education, 2009.

[38] S. Roncato, G. Sartori, J. Masterson and R. Rumiati, "Constructional apraxia: An information processing approach,” Cogn. Neuropsychol. vol. 4, pp. 113-29, 1987.

[39] G. Vallar, "The methodological foundations of neuropsychology," in Handbook of Clinical and Experimental Neuropsychology, G. Denes and L. Pizzamiglio, Eds. East Sussex, UK: Psychology Press. 1999, pp. 95-131.

[40] H. Damasio, and A. R. Damasio, Lesion Analysis in Neuropsychology, New York: Oxford University Press, 1989.

[41] R. Rosenthal and K. L. Fode, "Psychology of the scientist: V. Three experiments in experimenter bias," Psychol. Rep. vol. 12, pp. 491-511, 1963.

[42] G. Gainotti, "Constructional apraxia," in Handbook of Clinical Neurology - Volume 1, J. A. Frederiks Ed. Amsterdam: Elsevier, 1985, pp. 491-506.

[43] D. Wechsler, Wechsler Adult Intelligence Scale - Fourth Edition, San Antonio, TX: Psychological Corporation, 2008.

[44] D. Wechsler, Wechsler Memory Scale - Fourth Edition: Technical and Interpretive Manual, San Antonio, TX: Psychological Corporation, 2009. 
[45] NCS Pearson. Advanced Clinical Solutions for WAIS-IV and WMS-IV: Administration and Scoring Manual. San Antonio, TX: Psychological Corporation, 2009.

[46] S. McCrea and T. Robinson, "Visual puzzles, figure weights and cancellation: Some preliminary hypotheses on the functional and neural substrates of these three new WAIS-IV subtests," ISRN Neurology. vol. 2011, Article ID 123173, 19 pages, 2011. doi: 10.5402/2011/123173.

[47] R. A. McCarthy and E. K. Warrington, Cognitive Neuropsychology: A Clinical Introduction. San Diego, CA: Academic Press, 1990.

[48] E. W. Russell, "A reference method for constructing neuropsychological test batteries," J. Clin. Exp. Neuropsychol. vol. 9, pp. 376-392, 1987.

[49] L. C. Robertson, R. T. Knight, R. Rafal and A. P. Shimamura, "Cognitive neuropsychology is more than single-case studies," J. Exp. Psychol. Learn. vol. 19: pp. 710-17, 1993.

[50] Philips Healthcare. [Philips iSite Enterprise]. Andover, MA. K.

[51] H. J. Kretschmann and W. Weinrich, Eds. Cranial Neuroimaging and Clinical Neuroanatomy: Atlas of MR Imaging and Computed Tomography - Third Edition. New York: Thieme, 2001

[52] F. I. Craik and R. S. Lockhart, "Levels of processing: A framework for memory research," J. Verb. Learn. Verb. Beh. vol. 11, pp. 671-684, 1972.

[53] D. E. Nee, J. Jonides and M. J. Berman, "Neural mechanisms of proactive interference resolution," NeuroImage. vol. 38, pp. 740-51, 2007.

[54] D. Farias, C. Davis and G. Harrington, "Drawing: Its contribution to naming in aphasia," Brain Lang. vol. 97, pp. 53-63, 2006.

[55] N. A. DeFilipis and E. McCampbell, The Booklet Category Test: Second Edition - Professional Manual, Odessa, FL: Psychological Assessment Resources, Inc, 1997.

[56] E. K. Warrington and M. James, The Visual Object and Space Perception Battery. London, UK: Pearson Assessment, 1991.

[57] E. K. Warrington and M. James, "A new test of object decision: 2D silhouettes featuring a minimal view," Cortex, vol. 27, pp. 370-383, 1991.

[58] R. K. Heaton, G. J. Chelune, J. L. Talley, G. G. Kay and G. Curtiss, Wisconsin Card Sorting Test Manual: Revised and Expanded. Odessa, FL: Psychological Assessment Resources, Inc. 1993.

[59] D. T. Stuss, B. Levine, M. P. Alexander et al. Wisconsin Card Sorting Test performance in participants with focal frontal and posterior brain damage: Effects of lesion location and test structure on separable cognitive processes. Neuropsychologia, vol. 38 , pp. $388-402,2000$.

[60] C. G. Matthews and K. Klove, Instructional Manual for the Adult Neuropsychological Test Battery, Madison, WI: University of Wisconsin Medical School, 1964.

[61] C. Randolph, Repeatable Battery for the Assessment of Neuropsychological Status. San Antonio, TX: Psychological Corporation, 1998.
[62] D. Schretlen, Brief Test of Attention: Professional Manual. Odessa, FL: Psychological Assessment Resources, Inc, 1997.

[63] M. R. Trenerry, B. Crosson, J. Deboe and W. R. Leber, Visual Search and Attention Test: Professional Manual. Odessa, FL: Psychological Assessment Resources, Inc, 1990.

[64] R. M. Ruff, Ruff Figural Fluency Test: Professional Manual. Odessa, FL: Psychological Assessment Resources, Inc, 1996.

[65] M. A. Peterson and G. Rhodes Eds. Perception of Faces, Objects and Scenes: Analytic and Holistic Processes. New York: Oxford University Press, 2003.

[66] B. Stemmer, "Neuropragmatics: Disorders and Neural Systems," in Handbook of the Neuroscience of Language, B. Stemmer and H.A. Whitaker, Eds. New York: Academic Press, 2008, pp. 175-187.

[67] L. Pisella, N. Alahyane, A. Blangero et al. "Right-hemisphere dominance for visual remapping in humans," Philos. T. Roy. Soc. B. vol. 366, pp. 572-85, 2011.

[68] S. McCrea, L. Buxbaum, and B. Coslett, "Illusory conjunctions in simultanagnosia: Coarse coding of visual feature location?" Neuropsychologia, vol. 44, pp. 1724-36, 2006.

[69] R. Cabeza and A. Kingstone, Eds. The Handbook of Functional Neuroimaging - Second Edition, Cambridge, MA: MIT Press, 2006

[70] D. C. Delis, E. Kaplan and J. E. Kramer, Delis-Kaplan Executive Functions System. San Antonio, TX: Psychological Corporation, 2001.

[71] A. Kertesz, The Western Aphasia Battery - Revised. San Antonio, TX: Psychological Corporation, 2006.

[72] D. P. Roeltgen, "Agraphia." in Clinical Neuropsychology Fourth Edition, K. Heilman and E. Valenstein, Eds. New York: Oxford University Press, 2003, pp. 126-145.

[73] J. G. Beaumont, P. Kenealy and M. Rogers et al. Eds. The Blackwell Dictionary of Neuropsychology. Oxford, UK: Blackwell Publishers, 1999.

[74] H. B. Coslett and E. M. Saffran, "Optic aphasia and the right hemisphere: A replication and extension," Brain Lang. vol. 43, pp. 148-161, 1992.

[75] R. Reitan and D. Wolfson, The Halstead-Reitan Neuropsychological Test Battery: Theory and Interpretation, Tucson, AZ: Neuropsychology Press, 1993.

[76] R. Vocat, F. Staub, T. Stroppini and P. Vuilleumier, "Anosagnosia for hemiplegia: A clinical-anatomical prospective study," Brain, vol. 133, pp. 3578-97, 2010.

[77] A. L. Benton, K. Hamsher and A. B. Sivan, Multilingual Aphasia Examination - Third Edition, Odessa, FL: Psychological Assessment Resources, Inc, 1994.

[78] G. Denes and L. Pizzamiglio, Handbook of Clinical and Experimental Neuropsychology. East Sussex, UK: Psychology Press, 1999.

[79] L. F. D'Elia, P. Satz, C. Lyons-Uchiyama and T. White, Color Trails Test. Odessa, FL: Psychological Assessment Resources, Inc., 1996.

[80] D. Wechsler, Wechsler Adult Intelligence Scale - Third Edition. San Antonio, TX: Psychological Corporation, 1997. 
[81] D. Wechsler, Wechsler Memory Scale - Third Edition. San Antonio, TX: Psychological Corporation, 1997.

[82] A. G. Canavan, R. E. Passingham, C. D. Marsden et al. "Sequencing ability in Parkinsonian participants with frontal lobe lesions and participants who have undergone unilateral temporal lobectomies," Neuropsychologia, vol. 27, pp. 787-98, 1989.

[83] C. E. Curtis and M. D'Esposito, "Functional neuroimaging of working memory," in Handbook of Functional Neuroimaging Second Edition, R. Cabeza and A. Kingstone Eds. Cambridge, MA: MIT Press, 2006, pp. 269-306.

[84] C. A. Marzi, "Neuropsychology of attention" in Handbook of Clinical and Experimental Neuropsychology. G. Denes and L. Pizzamiglio, Eds. East Sussex, UK: Psychology Press, 1999, pp. 509-524.

[85] W. C. Culbertson and E. A. Zillmer, Tower of London - Drexel University: Second Edition. Toronto, ON: Multi-Health Systems, Inc, 2005.

[86] S. McCrea, "A functional magnetic resonance imaging study of the body schema using full human line-drawing figures in an on-line verbal naming and localization task of single body part words,” Behav. Brain Res. vol. 180, pp. 235-240, 2007.

[87] G. Denes, J. Y. Cappelletti, T. Zilli, F. Dalla Porta and A. Gallana, "A category-specific deficit of spatial representation: The case of autotopagnosia," Neuropsychologia, vol. 38, pp. 345-350, 2000.

[88] G. S. Wilkinson and G. J. Robertson, WRAT-4: Wide Range Achievement Test - Professional Manual, Lutz, FL: Psychological Assessment Resources, Inc., 2008.

[89] H. Shim, R. S. Hurley, E. Rogalski et al. "Anatomic, clinical and neuropsychological correlates of spelling errors in primary progressive aphasia," Neuropsychologia, vol. 50, pp. 1929-35, 2012.

[90] M. P. Kirschen et al. "Load- and practice-dependent increases in cerebro-cerebellar activation in verbal working memory: An fMRI study," NeuroImage, vol. 24, pp. 462472, 2005.

[91] L. De Witte, R. Brouns, D. Kavadias, S. Engelborghs, P. P. De Deyn and P. Marien, "Cognitive, affective and behavioral disturbances following vascular thalamic lesions: A review," Cortex, vol. 47, pp. 273-319, 2011.

[92] L. De Witte, J. Verhoeven, S. Engelborghs, P. P. De Deyn, and P. Marien, "Crossed aphasia and visuo-spatial neglect following right thalamic stroke: A case study and review of the literature," Behav. Neurol. vol. 19, pp. 177-194, 2008.

[93] K. Yamada, Y. Nagakane, K. Yoshikawa et al. "Somatotopic organization of thalamocortical projection fibers as assessed by MR tractography," Radiology, vol. 242, pp. 840-845, 2007.

[94] J. Schwoebel and H. B Coslett, "Evidence of multiple distinct representations of the human body," J. Cogn. Neurosci. vol. 17, pp. 543-553, 2005.

[95] H. O. Karnath and B. Baier, "Right insula for our sense of limb ownership and self-awareness of actions," Brain Struct. Funct. vol. 214, pp. 411-17, 2010.

[96] A. D. Smith, "On the use of drawing tasks in neuropsychological assessment," Neuropsychology, vol. 23, pp. 231-39, 2009 .

[97] M. J. Riddoch, "Loss of visual imagery: A generation deficit," Cogn. Neuropsychol. vol. 7, pp. 249-273, 1990.

[98] D. Grossi, A. Orsini, A. Modafferi and M. Liotti, "Visuoimaginal constructional apraxia: On a case of selective deficit in imagery," Brain Cogn. vol. 5, pp. 255-267, 1986.

[99] L. Trojano and D. Grossi, "A critical review of mental imagery deficits?” Brain Cogn. vol. 24, pp. 213-243, 1994.

[100] J. R. Booth, D. D. Burman, J. R. Meyer et al. "Functional anatomy of intra- and cross-modal lexical tasks," NeuroImage, vol. 16, pp. 7-22, 2002.

[101] J. R. Booth, D. D. Burman, J. R. Meyer et al., "Relation between brain activation and lexical performance," Hum. Brain Mapp. vol. 19, pp. 155-169, 2003.

[102] L. Cohen, A. Jobert, D. Le Bihan and S. Dehaene, "Distinct unimodal and multimodal regions for word processing in the left temporal cortex," NeuroImage, vol. 23, pp. 1256-1270, 2004.

[103] S. L. Thompson-Schill, I. P. Kan and R. T. Oliver, "Functional neuroimaging of semantic memory" in Handbook of Functional Neuroimaging - Second Edition, R. Cabeza and A. Kingstone Eds. Cambridge, MA: MIT Press, 2006, pp. 149-190.

[104] S. Epelbaum, P. Pinel, R. Gaillard et al. "Pure alexia as a disconnection syndrome: New diffusion imaging evidence for an old concept." Cortex, vol. 44, pp. 962-974, 2008.

[105] B. L. Miller, J. Cummings, F. Mishkin et al. "Emergence of artistic talent in frontotemporal dementia," Neurology, vol. 51, pp. 978-982, 1998.

[106] M. F. Schwartz, D. Y. Kimberg, G. M. Walker et al. "Neuroanatomical dissociation for taxonomic and thematic knowledge in the human brain," Proc. Natl. Acad. Sci. USA. vol. 108, pp. 8520-24, 2011.

[107] E. Mandonnet, A. Nouet, P. Gatignol, L. Capelle, and H. Duffau, "Does the left inferior longitudinal fasciculus play a role in language? A brain stimulation study," Brain, vol. 130, pp. 623-29, 2007.

[108] M. Catani and D. H. ffytche, "The rises and falls of disconnection syndromes," Brain, vol. 128, 2224-2239, 2005.

[109] M. Chechlacz, A. Novick, P. Rotshtein, W-L. Bickerington, G.W. Humphreys and N. Demeyere, "The neural substrates of drawing: A voxel-based morphometry analysis of constructional, hierarchical and spatial representation deficits," J. Cogn. Neurosci. vol. 26, pp. 2701-15, 2014.

[110] A. D. Milner and M. A. Goodale, The Visual Brain in Action: Second Edition. New York: Oxford University Press, 2006. 